\title{
A fault feature extraction method for single-channel signal of rotary machinery based on VMD and KICA
}

\author{
Hongchun Sun ${ }^{1}$, Liang Fang ${ }^{2}$, Feng Zhao ${ }^{3}$ \\ School of Mechanical Engineering and Automation, Northeastern University, Shenyang, China \\ Key Laboratory of Vibration and Control of Aero-Propulsion Systems of Ministry of Education, \\ Northeastern University, Shenyang, China \\ ${ }^{1}$ Corresponding author \\ E-mail: ${ }^{1}$ hchsun@mail.neu.edu.cn, ${ }^{2}$ shine_xiaoliang@126.com, ${ }^{3}$ wfjmqquh@hotmail.com
}

Received 11 July 2018; received in revised form 3 October 2018; accepted 16 October 2018 DOI https://doi.org/10.21595/jve.2018.20073

Check for updates

Copyright $₫ 2019$ Hongchun Sun, et al. This is an open access article distributed under the Creative Commons Attribution License, which permits unrestricted use, distribution, and reproduction in any medium, provided the original work is properly cited.

\begin{abstract}
A feature extraction method combined with variational mode decomposition (VMD) and kernel independent component analysis (KICA) is proposed to improve the fault feature extraction of vibration signal of rotary machinery. Firstly, VMD is used to decompose the single-channel vibration signal. Secondly, calculate the correlation coefficient between each component and the original signal. Finally, a new multidimensional observation signal is formed with high correlation components, and the fault signals will be extracted from the new observation signal by KICA. Compared with some typical fault feature extraction methods, the better performance of the proposed method is demonstrated by two experiments which are faulty rolling bearing experiment and a comprehensive experiment with faulty rolling bearing and faulty gear. Furthermore, an experiment of faulty rotary shaft verifies the effectiveness of this method. The results demonstrate that the proposed method is efficient for fault feature extraction of single-channel vibration signal of rotary machinery.
\end{abstract}

Keywords: feature extraction, single-channel vibration signal, rotary machinery, VMD, KICA.

\section{Introduction}

Because of the progress of science and technology, rotary machinery is becoming more and more complex and precise in order to produce products of higher quality and higher precision. However, what follows is the complexity of its fault diagnosis. From the earliest dismantling to today's intelligent diagnosis, fault diagnosis has been studied for decades. From an empirical point of view, the fault of rotary machinery usually occurs in bearings, gears and rotary shafts. Therefore, many researchers are studying the fault diagnosis of these components by extracting fault features of vibration signals recently [1-3]. During the acquisition of vibration signals, due to a limited monitoring environment, it is often single-channel monitoring. In order to acquire more fault information of single-channel vibration signals of rotary machinery and improve the efficiency of fault diagnosis, many feature extraction methods have been proposed, some typical methods such as wavelets [4, 5], ensemble empirical mode decomposition (EEMD) + fast independent component analysis (FastICA) $[6,7]$ and so on. However, these methods have the problem that the extracted features sometimes are not obvious, and the noise energy is very high.

EEMD [8] is widely used in fault feature extraction, but it sometimes has mode mixing. Furthermore, it is affected by the sampling frequency, and the decomposition error sometimes is large. In order to avoid these problems, a new adaptive signal processing method, VMD $[9,10]$, is proposed. This method is used to search the optimal solution of the variational model by iterative search to determine the frequency center and bandwidth of each decomposition component. FastICA [11, 12] and KICA [13, 14] which are blind source separation (BSS) algorithms are often used in fault diagnosis recently. The vibration signal of large rotary machinery often mixes several signals and shows a strong nonlinear characteristic. Compared with FastICA, KICA has the advantage of extracting nonlinear signals. Therefore, this paper proposes a fault feature extraction method combining the advantage of VMD and KICA. 
In this paper, a fault feature extraction method of single-channel vibration signal based on VMD-based KICA is proposed. The three experiments show that this method has a better performance than wavelets and EEMD + FastICA to extract the fault features of rotary machinery. The structure of this paper is as follows: the theory of VMD, KICA, correlation coefficient and evaluation index $\rho$ are introduced in Section 2. Section 3 gives a detailed procedure of the proposed method. The performance of the proposed method with faulty rolling bearing is evaluated in Section 4.1. In Section 4.2, the performance of the proposed method is evaluated by a comprehensive experiment with faulty rolling bearing and faulty gear. In Section 4.3, an experiment of faulty rotary shaft proves the effectiveness of the proposed method.

\section{Theories}

\subsection{VMD}

VMD is a new signal decomposition method, which is the process of solving the variational problem based on classical Wiener filter, Hilbert transform and frequency mixing. In this method, the frequency center and bandwidth of each decomposition component are determined by searching the optimal solution of the variational model, and the signal can be adaptively decomposed into a component with sparsity. It is assumed that each mode is a limited bandwidth with a central frequency, the central frequency and bandwidth are constantly updated during the decomposition process, and VMD is finding the mode function $f_{k}(t), k \in\{1,2, \cdots, K\}$ with the smallest sum of $K$ estimation bandwidths, and the sum of the mode functions is the input signal $S$. The bandwidth of each mode function is determined through the following steps:

(1) In order to obtain the analytic signal of the mode function, Hilbert transform is performed for each mode function $f_{k}(t)$, that is:

$\left[\delta(t)+\frac{j}{\pi t}\right] f_{k}(t)$

where $t$ is time, $\delta(t)$ is impact function, $\left\{f_{k}(t)\right\}=\left\{f_{1}(t), f_{2}(t), \cdots, f_{K}(t)\right\}$ is $K$ components.

(2) Modulate the spectrum of each mode to the corresponding baseband, that is:

$\left[\left(\delta(t)+\frac{j}{\pi t}\right) f_{k}(t)\right] e^{-j \omega_{k} t}$

where $\left\{\omega_{k}\right\}=\left\{\omega_{1}, \cdots, \omega_{K}\right\}$ is the center frequency of $f_{k}(t)$.

(3) Estimate the bandwidth of each mode component. The corresponding constraint variational model expression is as follows:

$$
\left\{\begin{array}{l}
\min _{\left\{f_{k}(t)\right\},\left\{\omega_{k}\right\}}\left(\left\|\frac{\partial\left[\left(\delta(t)+\frac{j}{\pi t}\right) f_{k}(t)\right]}{\partial t} e^{-j \omega_{k} t}\right\|_{2}^{2}\right), \\
\sum_{k=1}^{K} f_{k}(t)=S(t) .
\end{array}\right.
$$

In order to obtain the above constraint variational problems, the penalty factor $\alpha$ of the quadratic term and the Lagrange multiplication operator $\lambda(t)$ are introduced. $\alpha$ is a large enough positive number, which can guarantee the reconstruction precision of the signal under the presence of Gaussian noise. The Lagrange operator makes the constraint conditions strict, and the extended Lagrange expression is: 


$$
\begin{aligned}
& L\left(\left\{f_{k}(t)\right\},\left\{\omega_{k}\right\}, \lambda(t)\right)=\alpha \sum_{k=1}^{K}\left\|\frac{\partial\left[\left(\delta(t)+\frac{j}{\pi t}\right) f_{k}(t)\right]}{\partial t} e^{-j \omega_{k} t}\right\|_{2}^{2} \\
& +\left\|S(t)-\sum_{k=1}^{K} f_{k}(t)\right\|_{2}^{2}+\left\langle\lambda(t), S(t)-\sum_{k=1}^{K} f_{k}(t)\right\rangle .
\end{aligned}
$$

The above variational problem is obtained by using alternate direction method of multipliers (ADMM), and the saddle points of the extended Lagrange expression can be obtained by alternately updating $f_{k}^{n+1}(t), \omega_{k}^{n+1}$ and $\lambda^{n+1}(t) . f_{k}^{n+1}(t)$ is the mode function at the $n+1$ th cycle, and $\omega_{k}^{n+1}$ is the center of the power spectrum of the current modal function. $\lambda^{n+1}(t)$ is the multiplication operator of the $n+1$ th cycle. The solution process of $f_{k}^{n+1}(t)$ can be expressed as:

$f_{k}^{n+1}(t)=\operatorname{argmin}\left\{\alpha\left\|\frac{\partial\left[\left(\delta(t)+\frac{j}{\pi t}\right) f_{k}(t)\right]}{\partial t} e^{-j \omega_{k} t}\right\|_{2}^{2}+\left\|S(t)-\sum_{i \neq k}^{K} f_{i}(t)+\frac{\lambda(t)}{2}\right\|_{2}^{2}\right\}$,

where $i \in\{1,2, \cdots, K\} \wedge i \neq k$. By using the Fourier isometric transformation, Eq. (5) can be transformed to the frequency domain and replaced $\omega$ with $\omega-\omega_{k}$, and the result is transformed into the form of negative frequency interval integral. The solution of the optimization problem is:

$\hat{f}_{k}^{n+1}(\omega)=\frac{\hat{S}(\omega)-\sum_{i<k} \hat{f}_{i}(\omega)-\sum_{i>k} \hat{f}_{i}(\omega)+\frac{\hat{\lambda}^{n}(\omega)}{2}}{1+2 \alpha\left(\omega-\omega_{k}\right)^{2}}, k \in\{1,2, \cdots, K\}$.

According to the same process, get the center frequency update mode:

$\omega_{k}^{n+1}=\frac{\int_{0}^{\infty} \omega\left|\hat{f}_{k}(\omega)\right|^{2} d \omega}{\int_{0}^{\infty}\left|\hat{f}_{k}(\omega)\right|^{2} d \omega}, \quad k \in\{1,2, \cdots, K\}$,

where $\hat{f}_{k}^{n+1}(\omega)$ is equivalent to the Wiener filtering of $\hat{S}(\omega)-\sum_{i \neq k} \hat{f}_{i}(\omega)$. The Fourier transform to $\left\{\hat{f}_{k}(\omega)\right\}$, the actual part is $\left\{f_{k}(t)\right\}$.

The specific implementation process of the VMD method is as follows:

(1) Initialize mode function $\left\{\hat{f}_{k}^{1}\right\}$, center frequency $\left\{\omega_{k}^{1}\right\}$, Lagrange multiplication operator, initial cycle times $n=0$.

(2) Execute the cycle $n=n+1$.

(3) Update $f_{k}^{n+1}, \omega_{k}^{n+1}$

(4) Update $\hat{\lambda}^{n+1}(\omega)$. $\hat{\lambda}^{n+1}(\omega)=\hat{\lambda}^{n}(\omega)+\tau\left(\hat{S}(\omega)-\sum_{k} \hat{f}_{k}^{n+1}(\omega)\right)$, where $\tau$ is a time constant, usually taken as 0 .

(5) Given discriminant accuracy $\varepsilon>0$, repeat the above steps until the iteration stop condition is satisfied:

$$
\sum_{k=1}^{K} \frac{\left\|\hat{f}_{k}^{n+1}(\omega)-\hat{f}_{k}^{n}(\omega)\right\|_{2}^{2}}{\left\|\hat{f}_{k}^{n}(\omega)\right\|_{2}^{2}}<\varepsilon
$$

\subsection{KICA}

The mathematical description of independent component analysis (ICA) is: $\mathbf{X}=\mathbf{A S}+\mathbf{N}$, where 
$\mathbf{S}=\left[s_{1}, s_{2}, \cdots, s_{N}\right]$ is a vector of $N$ source signals, $\mathbf{X}=\left[x_{1}, x_{2}, \cdots, x_{M}\right]$ is a vector of $\mathrm{M}$ mixed signals, The mixing matrix $\mathbf{A}$ is a $M \times N$ dimensional matrix, $\mathbf{N}$ is noise vector. The meaning of ICA is that when the mixing matrix and source signals are unknown, we only determine the removemixing matrix $\mathbf{W}$ based on the observed data, and the output $\widehat{\boldsymbol{S}}=\mathbf{W} \mathbf{X}$ is the estimation of source signals.

KICA is not a simple nucleation of ICA, but a new ICA method. The idea of kernel technology is to use nonlinear mapping $\phi: R^{m} \rightarrow R$, and map the nonlinear variable $y^{i} \in R^{m}(i=1,2, \cdots, N)$ of original input space into a kernel feature space $R$ to linearize it, and then analyse the mapped data in this feature space. Thus, the linear blind source separation in the space $R$ is equivalent to nonlinear blind source separation in the original space. One of the important characteristics of this technology is that kernel function can be used instead of inner product between two vectors to realize nonlinear transformation without specific form being considered. The kernel function of radial basis function (RBF) $K\left(x_{i}, y_{i}\right)=\exp \left(-\left\|x_{i}-y_{i}\right\| / \delta^{2}\right)$ is chosen here.

KICA is characterized by the use of nonlinear functions as a contrast function in reproducing kernel Hilbert space (RKHS), the signal is mapped from low-dimensional space to the high-dimensional space, and this method is used to find out the minimum value of contrast function in this space. This function has a certain correlation with mutual information and has better mathematical properties. Moreover, this function space is suitable for various source signals. Therefore, compared with traditional ICA, KICA has better flexibility and robustness.

The KICA's contrast function is constructed by calculating the correlation of a set of random variables directly. Let $F$ be a real vector function space, for simple calculations, $s_{1}$ and $s_{2}$ are two unary random variables of space $F$. Define the correlation coefficient $\tau_{F}$ of $s_{1}$ and $s_{2}$ as the maximum correlation coefficient between random variables $f_{1}\left(s_{1}\right)$ and $f_{2}\left(s_{2}\right)$ :

$\tau_{F}=\max _{f_{1}, f_{2} \in F} \operatorname{corr}\left(f_{1}\left(s_{1}\right), f_{2}\left(s_{2}\right)\right)=\max _{f_{1}, f_{2} \in F} \frac{\operatorname{cov}\left(f_{1}\left(s_{1}\right), f_{2}\left(s_{2}\right)\right)}{\left(\operatorname{var} f_{1}\left(s_{1}\right)\right)^{\frac{1}{2}}\left(\operatorname{var} f_{2}\left(s_{2}\right)\right)^{\frac{1}{2}}}$.

$\tau_{F}$ is also called a contrast function between random variables. Obviously, if $s_{1}$ and $s_{2}$ are independent, so, $\tau_{F}=0$. If space $F$ is large enough and $\tau_{F}=0, s_{1}$ and $s_{2}$ are also independent of each other. The procedure of KICA is as follows:

Input: Data vector $x_{1}, x_{2}, \ldots, x_{n}$ and kernel function $k(x, s)$.

Step 1: Whiten data vector $x_{1}, x_{2}, \ldots, x_{n}$.

Step 2: Use Cholesky decomposition to find the Gram matrix $\left(\mathbf{G}_{1}, \mathbf{G}_{2}, \ldots, \mathbf{G}_{m}\right)$ of original independent data, where $s_{i}=\mathbf{W} x_{i}(\mathbf{W}$ is the remove-mixing matrix).

Step 3: Define $\lambda_{H}$ be the maximum eigenvalue of Eq. (10):

$$
\left(\begin{array}{cccc}
\mathbf{G}_{1} \mathbf{G}_{1} & \mathbf{G}_{1} \mathbf{G}_{2} & \cdots & \mathbf{G}_{1} \mathbf{G}_{m} \\
\mathbf{G}_{2} \mathbf{G}_{1} & \mathbf{G}_{2} \mathbf{G}_{2} & \cdots & \mathbf{G}_{2} \mathbf{G}_{m} \\
\vdots & \vdots & \ddots & \vdots \\
\mathbf{G}_{m} \mathbf{G}_{1} & \mathbf{G}_{m} \mathbf{G}_{2} & \cdots & \mathbf{G}_{m} \mathbf{G}_{m}
\end{array}\right)\left(\begin{array}{c}
\beta_{1} \\
\beta_{2} \\
\vdots \\
\beta_{m}
\end{array}\right)=\lambda\left(\begin{array}{cccc}
\mathbf{G}_{1} \mathbf{G}_{1} & 0 & \cdots & 0 \\
0 & \mathbf{G}_{2} \mathbf{G}_{2} & \cdots & 0 \\
\vdots & \vdots & \ddots & \vdots \\
0 & 0 & \cdots & \mathbf{G}_{m} \mathbf{G}_{m}
\end{array}\right)\left(\begin{array}{c}
\beta_{1} \\
\beta_{2} \\
\vdots \\
\beta_{m}
\end{array}\right) .
$$

Step 4: Minimize $M_{\lambda_{H}}=-0.5 \log \lambda_{H}$ for $\mathbf{W}$.

Output: W.

This algorithm keeps running repeatedly between Step 2 and Step 4 until the convergence condition is satisfied, so that the remove-mixing matrix $\mathbf{W}$ can be obtained. According to $s=\mathbf{W} x$, for a set of observed data $x_{1}, x_{2}, \ldots, x_{n}$, the original independent source signals can be estimated effectively through the remove-mixing matrix $\mathbf{W}$.

\subsection{Correlation coefficient}

The formula for the correlation coefficient $\sigma_{x y_{i}}$ of component signal to the source signal is as 
follows:

$\sigma_{x y_{i}}=\frac{\operatorname{cov}\left(\mathbf{X}, \mathbf{Y}_{i}\right)}{\sqrt{D(\mathbf{X})} \sqrt{D\left(\mathbf{Y}_{i}\right)}}$,

where $\mathbf{X}$ is the mixed source signal. $\mathbf{Y}_{i}$ is the $i$ th component signal. $D(\mathbf{X})$ and $D\left(\mathbf{Y}_{i}\right)$ are the variance of signal $\mathbf{X}$ and $\mathbf{Y}_{i} \cdot \operatorname{cov}\left(\mathbf{X}, \mathbf{Y}_{i}\right)$ is the covariance between the signal $\mathbf{X}$ and $\mathbf{Y}_{i}$.

\subsection{Evaluation index $\rho$}

The fault signal extracted from the real signal generally contains some noise. In the power spectrum of the extracted fault signal, the power of the fault frequency is usually the highest, so other frequencies can be considered as noise. If the power of noise is very close to or even higher than the power of fault frequency, the fault type cannot be accurately determined. Therefore, in order to compare the extraction effect of each fault diagnosis method, this paper proposes an evaluation index $\rho$ :

$\rho=\frac{P_{f}-P_{s}}{P_{s}}$

where $P_{f}$ is the power of fault frequency. $P_{S}$ is the power of the noise of the highest amplitude. Therefore, the bigger the value of $\rho$, the better the effect of the extraction.

\section{Detailed procedure of the proposed method}

In this paper, a fault diagnosis method of rotary machinery based on VMD-based KICA is proposed. The procedure of the proposed method has been summarized in Fig. 1. The detailed procedure is described as follows:

Step 1: The collected single-channel signal $\mathbf{X}(t)$ is decomposed with VMD and each component signal can be obtained.

Step 2: Calculate the correlation coefficient of each component signal to $\mathbf{X}(t)$. Then, choose high correlation component signals to form a new observation signal.

Step 3: Use KICA to extract the fault signals from the new observation signal.

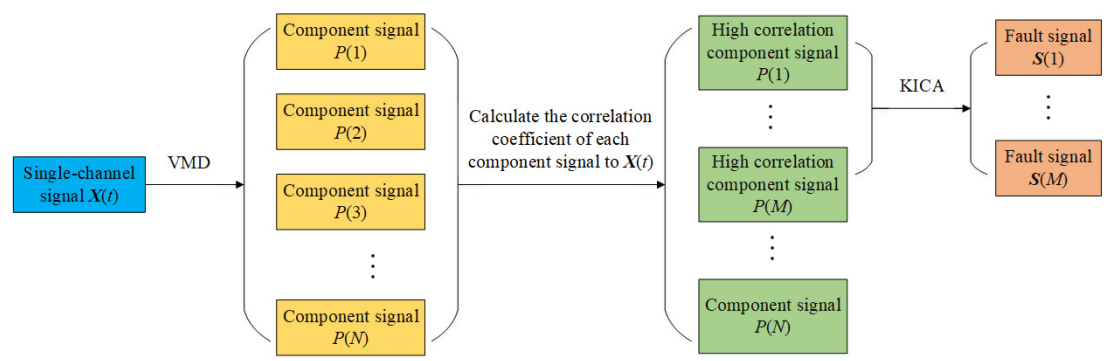

Fig. 1. The summarized procedure of the proposed method

\section{Experiments}

\subsection{Faulty rolling bearing experiment}

In order to verify the validity of the proposed method for faulty rolling bearing vibration signal, the test data from the bearing database of Case Western Reserve University are selected for analysis. The rolling bearing used in the test is SKF6205. The motor speed is $1800 \mathrm{rpm}$, that is, the rotation frequency $f_{r}=30 \mathrm{~Hz}$. The sampling frequency $f_{s}=12000 \mathrm{~Hz}$ and the number of 
sampling points is 12000 . The inner and outer rings of the rolling bearing are respectively machined with tiny pitting pits to simulate the fault of inner ring and outer ring. The fault frequency of inner ring can be expressed as:

$f_{i c}=\frac{1}{2} N\left(1+\frac{d}{D} \cos \alpha\right) f_{r}$

The fault frequency of outer ring can be expressed as:

$f_{o c}=\frac{1}{2} N\left(1-\frac{d}{D} \cos \alpha\right) f_{r}$

where $N$ is the number of roller, $d$ is the diameter of roller, $D$ is the pitch diameter of bearing, and $\alpha$ is bearing contact angle. According to Eq. (13) and Eq. (14), $f_{i c}=162 \mathrm{~Hz}$ and $f_{o c}=107 \mathrm{~Hz}$.

The time-domain waveform and power spectrum of single-channel vibration signal of inner ring fault and outer ring fault mixed are shown in Fig. 2.

The fault features of inner ring and outer ring cannot be found from time-domain waveform and power spectrum in Fig. 2. Therefore, so as to verify the high efficiency of the proposed method, the effect of feature extraction of wavelets, EEMD+FastICA and the proposed method are compared as follows.
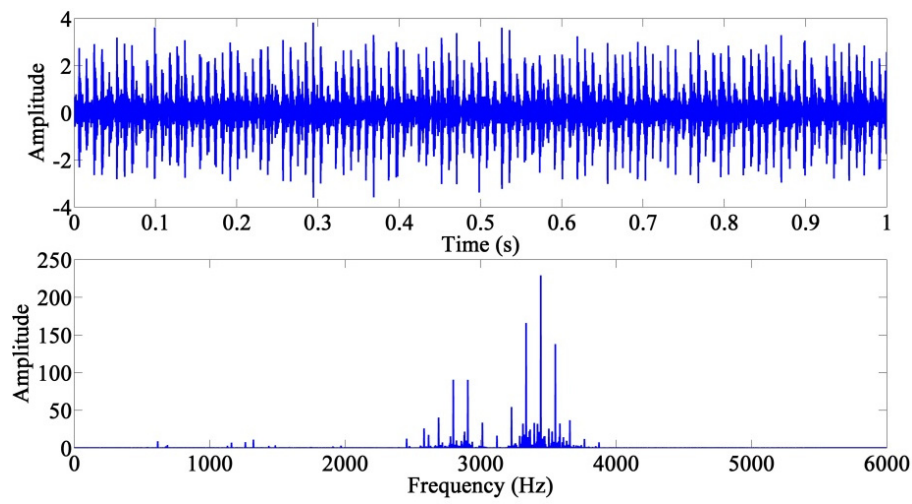

Fig. 2. The time-domain waveform and power spectrum of the observation signal

\section{A. Wavelets.}

In this method, the observation signal is decomposed with six-layer wavelet decomposition, and then the power spectrum of each component can be obtained. The result is shown in Fig. 3 . $f_{i c}$ and $f_{o c}$ are not extracted, so the evaluation index $\rho$ can be considered as $-\infty$. Therefore, wavelets cannot extract the feature of fault signals of rolling bearing.

B. EEMD + FastICA.

In this method, the observation signal is decomposed by EEMD first, and each intrinsic mode function (IMF) can be obtained. Then, calculate the correlation coefficient of each IMF to the observation signal. IMFs with high correlation coefficient were selected to reconstruct the new observation signal. Finally, the fault signals can be extracted by FastICA. The power spectrum of the extracted fault signals is shown in Fig. 4.

In Fig. 4(b), the frequency of $107.7 \mathrm{~Hz}$ which is very close to $f_{o c}$ is extracted and its double frequency is also extracted. The frequency of $538.3 \mathrm{~Hz}$ is the noise of the highest amplitude. The frequency of $161.9 \mathrm{~Hz}$ which is very close to $f_{i c}$ can be seen in Fig. 4(a), but the frequency of 107.7 Hz is also obvious, and it can be considered as noise now. Therefore, mode mixing occurs at this time. According to Eq. (12), $\rho_{o c}=4.687, \rho_{i c}=0.229$. 


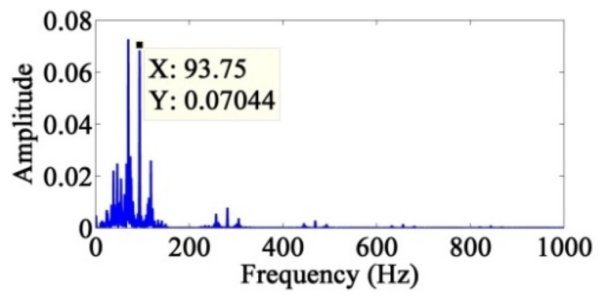

a)

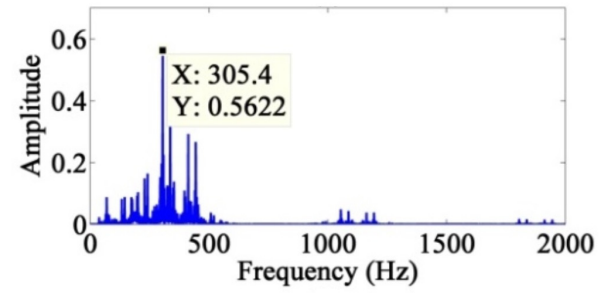

c)

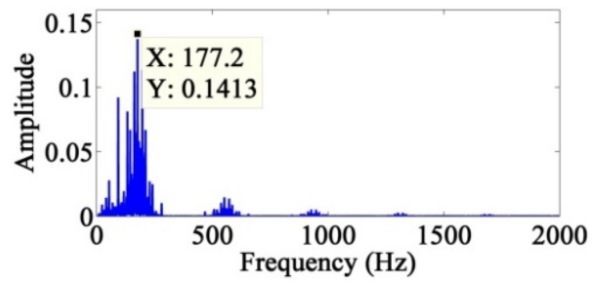

b)

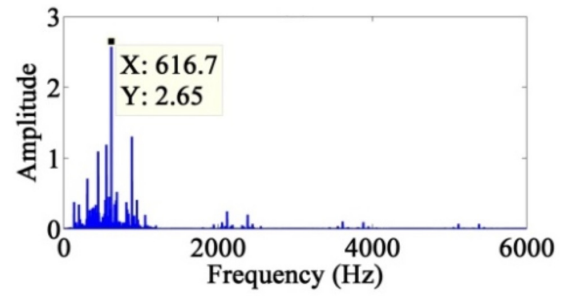

d)

Fig. 3. The power spectrum of the decomposed signals after wavelets: a) the sixth layer of low-frequency component, b) the sixth layer of high-frequency component, c) the fifth layer of high-frequency component, d) the fourth layer of high-frequency component

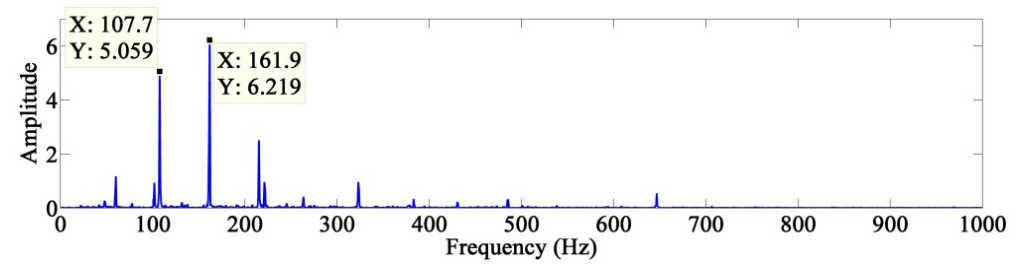

a)

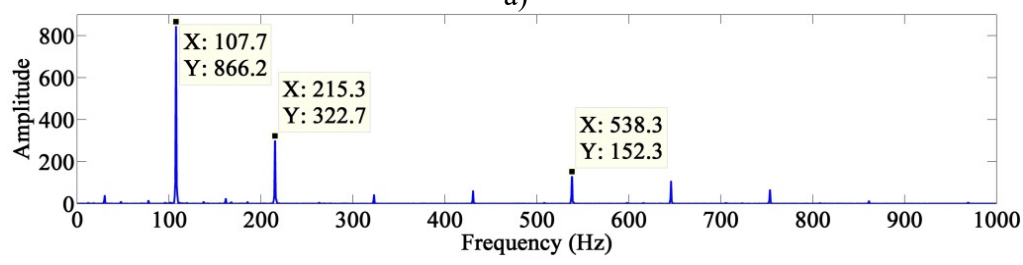

b)

Fig. 4. The power spectrum of the extracted signals after EEMD + FastICA

C. The proposed method.

In this method, the observation signal is decomposed by VMD $(K=4, \alpha=1000)$ first and each component signal can be obtained. Then, calculate the correlation coefficient of each component signal to the observation signal and their values are in Table 1. Component signals with high correlation coefficient (Threshold $>0.5$ ) were selected to reconstruct the new observation signal. Finally, the fault signals can be extracted by KICA. The power spectrum of the extracted fault signals is shown in Fig. 5.

The frequency of $161.9 \mathrm{~Hz}$ which is very close to $f_{i c}$ can be seen in Fig. 5(a). The frequency of $60.06 \mathrm{~Hz}$ is the noise of the highest amplitude. In Fig. 5(b), the frequency of $107.7 \mathrm{~Hz}$ which is very close to $f_{o c}$ is extracted and its double frequency is also extracted. The frequency of $30.03 \mathrm{~Hz}$ is the noise of the highest amplitude. Furthermore, the fault features are very obvious. According to Eq. (12), $\rho_{o c}=14.597, \rho_{i c}=1.046$. 
Table 1. Correlation coefficient of each component signal

\begin{tabular}{|c|c|}
\hline Serial number & Value \\
\hline $\mathrm{i} 1$ & 0.155 \\
\hline $\mathrm{i} 2$ & 0.193 \\
\hline $\mathrm{i} 3$ & 0.585 \\
\hline $\mathrm{i} 4$ & 0.801 \\
\hline
\end{tabular}
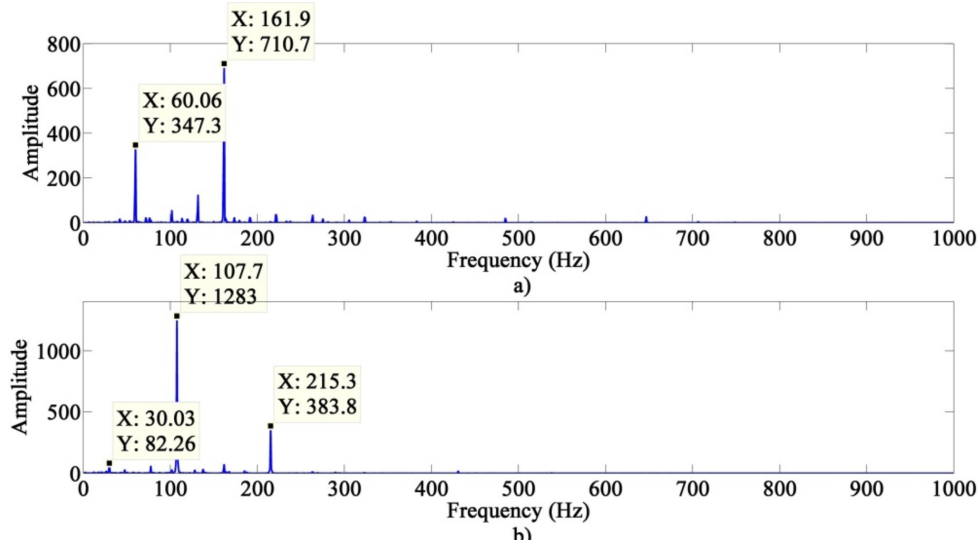

b)

Fig. 5. The power spectrum of the extracted signals after VMD + KICA

The evaluation index of the three methods are in Table 2. The $\rho$ of the proposed method is much bigger than other two methods. Therefore, the proposed method has a better effect of extraction. The result proves that the proposed method is efficient to extract the features of single-channel vibration signal of rolling bearing with the fault of inner ring and outer ring mixed.

Table 2. Evaluation index statistics

\begin{tabular}{|c|c|c|}
\hline Evaluation index & \multirow{2}{*}{$\rho_{o c}$} & $\rho_{i c}$ \\
\cline { 1 - 2 } Method & $-\infty$ & $-\infty$ \\
\hline Wavelets & $-\infty .687$ & 0.229 \\
\hline EEMD + FastICA & 4.597 & 1.046 \\
\hline
\end{tabular}

\subsection{Comprehensive experiment with faulty rolling bearing and faulty gear}

The experimental device is shown in Fig. 6. The whole device is driven by a $550 \mathrm{~W}$ $(220 \mathrm{~V}-50 \mathrm{~Hz}) \mathrm{AC}$ motor and drives the shaft system with couplings. The shaft section between two bearing seats is equipped with a belt wheel, and the belt drives the active gear shaft of gear box. One acceleration transducer is installed vertically on the shell near the rolling bearing. There are two rolling bearings on the shaft system, and one of them is machined with tiny pitting pits on the outer ring to simulate the fault of outer ring. The sampling frequency $f_{s}=8192 \mathrm{~Hz}$ and the number of sampling points is 8192 . The motor speed is $850 \mathrm{rpm}$, that is, the rotation frequency $f_{r}=14.2 \mathrm{~Hz}$.

According to Eq. (14), $f_{o c}=70 \mathrm{~Hz}$. Destroy one tooth of the driving gear to simulate the fault of tooth breaking. The number of gear teeth $Z=20$. The number of the broken teeth $Z_{b}=1$. According to $f_{c}=Z \times f_{r}$, the gear mesh frequency $f_{c}=283.3 \mathrm{~Hz}$. When the gear is affected by broken tooth fault, the rotation frequency $f_{r}$ and its frequency multiplication will be the main features in frequency-domain, and the frequency $f_{z}=Z_{b} \times f_{c}$ will also exist. The time-domain waveform and power spectrum of single-channel mixed signal are shown in Fig. 7. 


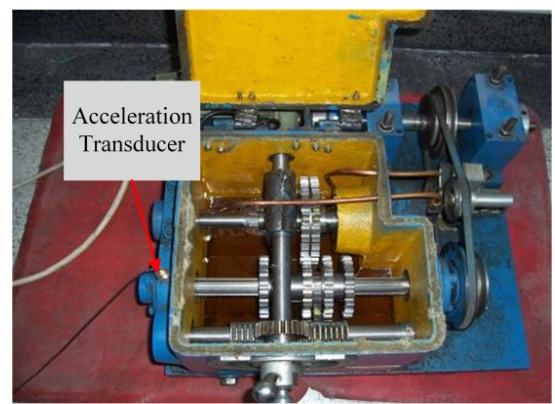

Fig. 6. The experimental device

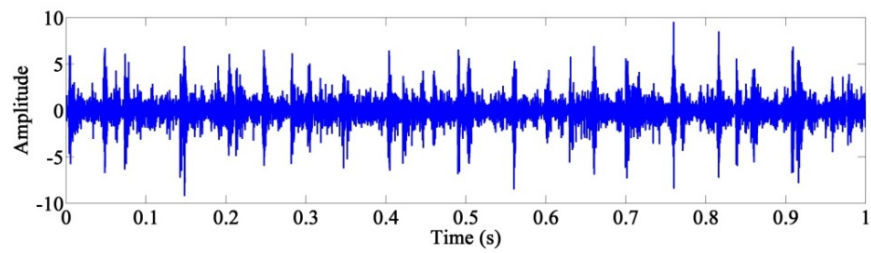

a)

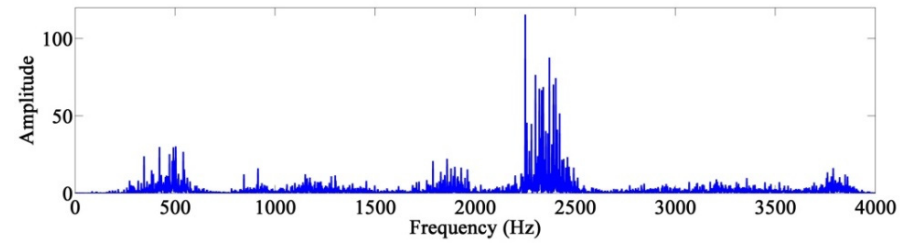

b)

Fig. 7. The time-domain waveform and power spectrum of the mixed signal

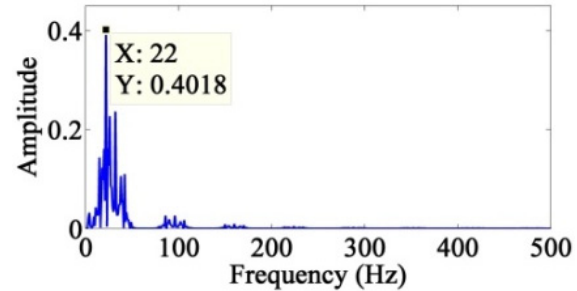

a)

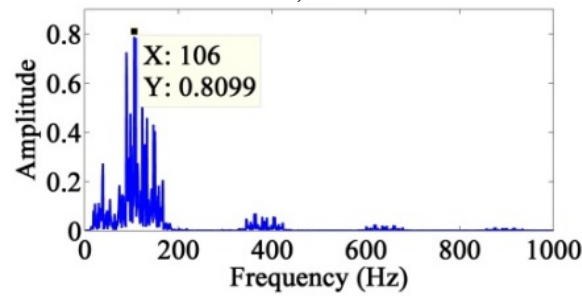

c)

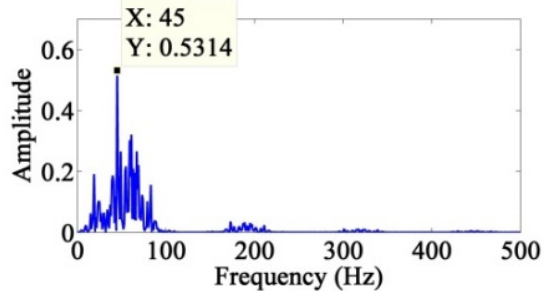

b)

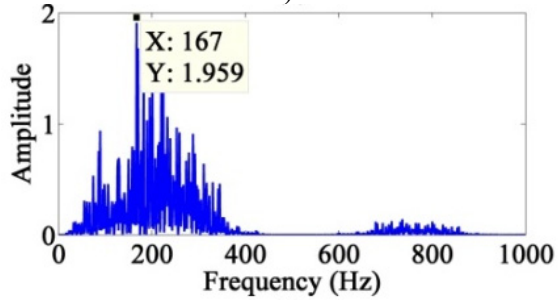

d)

Fig. 8. The power spectrum of the decomposed signals after wavelets: a) the seventh layer of low-frequency component, b) the seventh layer of high-frequency component, c) the sixth layer of high-frequency component, d) the fifth layer of high-frequency component

The fault features of outer ring and gear breaking cannot be found from time-domain waveform and power spectrum in Fig. 7. Therefore, the effect of feature extraction of wavelets, EEMD + FastICA and the proposed method are compared as follows:

A. Wavelets. 
In this method, the mixed signal is decomposed with seven-layer wavelet decomposition, and then the power spectrum of each component can be obtained. The result is shown in Fig. 8. The fault features are not extracted, so the evaluation index $\rho$ can be considered as $-\infty$.

B. EEMD + FastICA.

In this method, the mixed signal is decomposed by EEMD first, and each intrinsic mode function (IMF) can be obtained. Then, calculate the correlation coefficient of each IMF to the mixed signal. IMFs with high correlation coefficient were selected to reconstruct the new observation signal. Finally, the fault signals can be extracted by FastICA. The power spectrum of the extracted fault signals is shown in Fig. 9.

The frequency of $70 \mathrm{~Hz}$ which is very close to $f_{o c}$ can be seen in Fig. 9(a). The frequency of $20 \mathrm{~Hz}$ is the noise of the highest amplitude. In Fig. 9(b), the frequency of $14 \mathrm{~Hz}$ which is very close to $f_{r}$ and its double frequency are extracted, and the frequency $f_{c}$ is also extracted. The frequency of $72 \mathrm{~Hz}$ is the noise of the highest amplitude. According to Eq. (12), $\rho_{o c}=0.146$, $\rho_{g}=-0.220$.
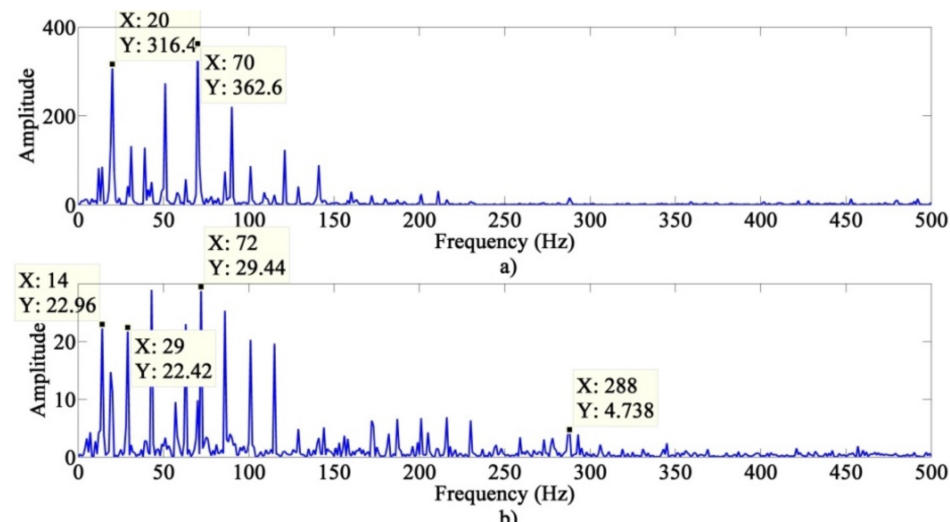

Fig. 9. The power spectrum of the extracted signals after EEMD + FastICA

C. The proposed method.

In this method, the mixed signal is decomposed by VMD $(K=6, \alpha=1000)$ first and each component signal can be obtained. Then, calculate the correlation coefficient of each component signal to the mixed signal and their values are in Table 3. Component signals with high correlation coefficient (Threshold $>0.5$ ) were selected to reconstruct the new observation signal. Finally, the fault signals can be extracted by KICA. The power spectrum of the extracted fault signals is shown in Fig. 10.
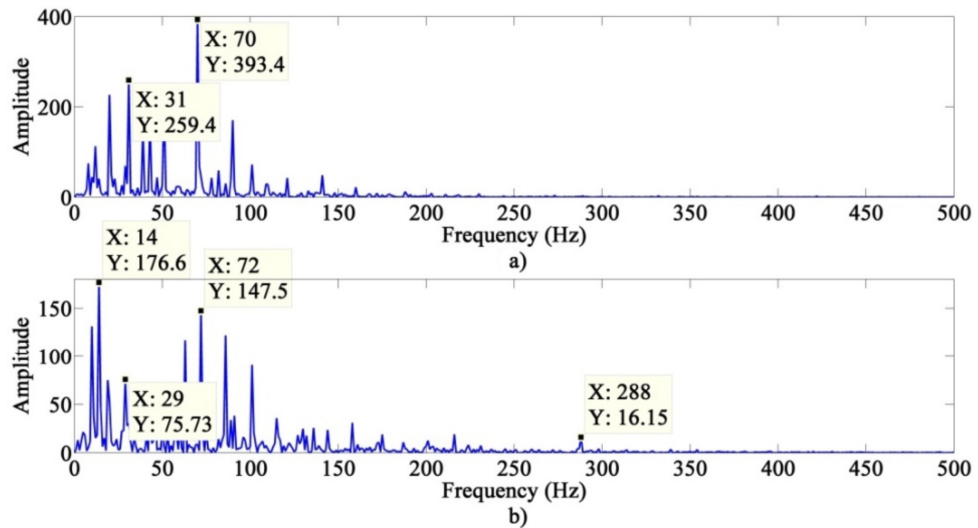

Fig. 10. The power spectrum of the extracted signals after VMD + KICA 
Table 3. Correlation coefficient of each component signal

\begin{tabular}{|c|c|}
\hline Serial number & Value \\
\hline i1 & 0.137 \\
\hline i2 & 0.150 \\
\hline i3 & 0.188 \\
\hline i4 & 0.606 \\
\hline i5 & 0.502 \\
\hline i6 & 0.091 \\
\hline
\end{tabular}

The frequency of $70 \mathrm{~Hz}$ which is very close to $f_{o c}$ can be seen in Fig. 10(a). The frequency of $31 \mathrm{~Hz}$ is the noise of the highest amplitude. In Fig. 10(b), the frequency of $14 \mathrm{~Hz}$ which is very close to $f_{r}$ and its double frequency are extracted, and the frequency $f_{c}$ is also extracted. The frequency of $72 \mathrm{~Hz}$ is the noise of the highest amplitude. Furthermore, the fault features are obvious. According to Eq. (12), $\rho_{o c}=0.517, \rho_{g}=0.197$.

The evaluation index of the three methods are in Table 4. The $\rho$ of the proposed method is much bigger than other two methods. Therefore, the proposed method has a better effect of extraction. The results demonstrate that the proposed method is efficient to extract the fault features of single-channel vibration signal of complex machinery.

Table 4. Evaluation index statistics

\begin{tabular}{|c|c|c|}
\hline Evaluation index & \multirow{2}{*}{$\rho_{o c}$} & $\rho_{g}$ \\
\hline Method & $-\infty$ & $-\infty$ \\
\hline Wavelets & $-\infty$ & -0.220 \\
\hline EEMD + FastICA & 0.146 & -197 \\
\hline The proposed method & 0.517 & 0.197 \\
\hline
\end{tabular}

\subsection{Faulty rotary shaft experiment}

A faulty rotary shaft experiment is applied to verify the effectiveness of the proposed method. The schematic diagram of test system is Fig. 11 and the test rig and signal acquisition system is shown in Fig. 12.

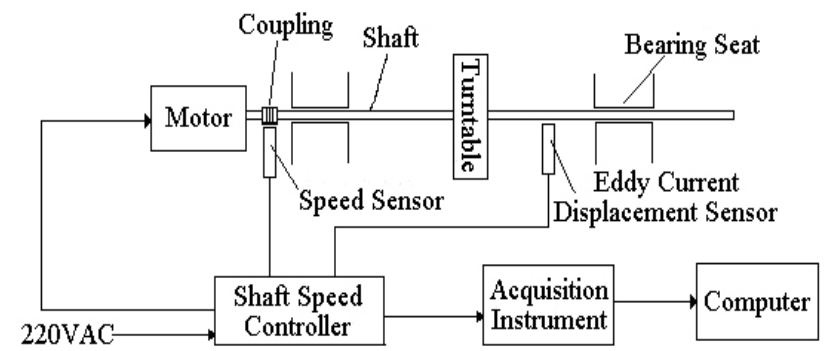

Fig. 11. The schematic diagram of test system

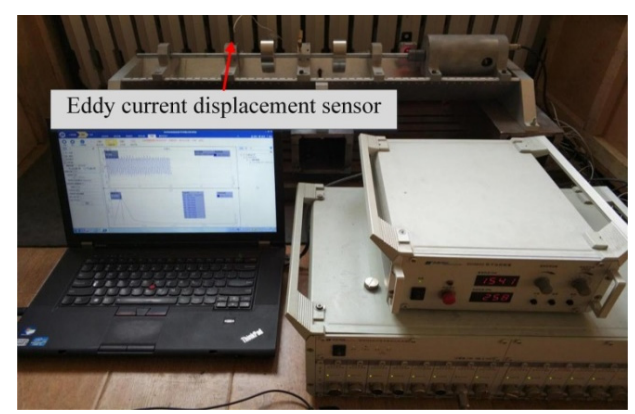

Fig. 12. The test rig and signal acquisition system of faulty rotary shaft 
In this experiment, the artificial misalignment fault and rub-impact fault are created. Only one eddy current displacement sensor is used to collect the mixed vibration signal of faulty shaft. The sampling frequency is $1000 \mathrm{~Hz}$ and the number of sampling points is 5000 . The motor speed is $2000 \mathrm{rpm}$, that is, the rotation frequency $f_{r}=33.3 \mathrm{~Hz}$. According to experience, the feature of misalignment fault is that a higher amplitude of $2 \times f_{r}$ appears, even exceeding the amplitude of $f_{r}$ in frequency-domain. The feature of rub-impact fault is emerging $1 / n$ of $f_{r}$, where $n$ is equal to 2, 3, or 4. The time-domain waveform of the collected signal is shown in Fig. 13.

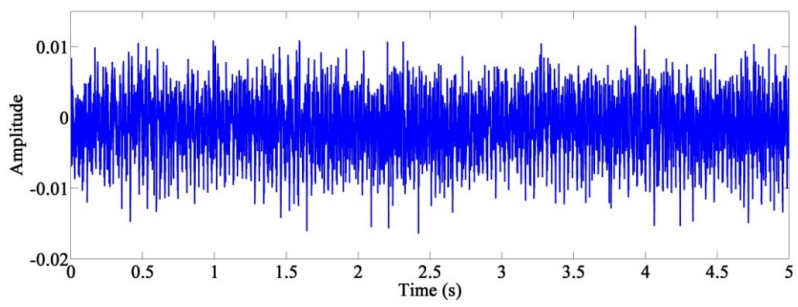

Fig. 13. The time-domain waveform of the collected signal

In the proposed method, the collected signal is decomposed by VMD $(K=4, \alpha=1000)$ first and each component signal can be obtained. Then, calculate the correlation coefficient of each component signal to the collected signal and their values are in Table 5. Component signals with high correlation coefficient (Threshold $>0.5$ ) were selected to reconstruct the new observation signal. Finally, the fault signals can be extracted by KICA. The power spectrum of the extracted fault signals is shown in Fig. 14.

Table 5. Correlation coefficient of each component signal

\begin{tabular}{|c|c|}
\hline Serial number & Value \\
\hline i1 & 0.551 \\
\hline i2 & 0.579 \\
\hline i3 & 0.135 \\
\hline i4 & 0.133 \\
\hline
\end{tabular}
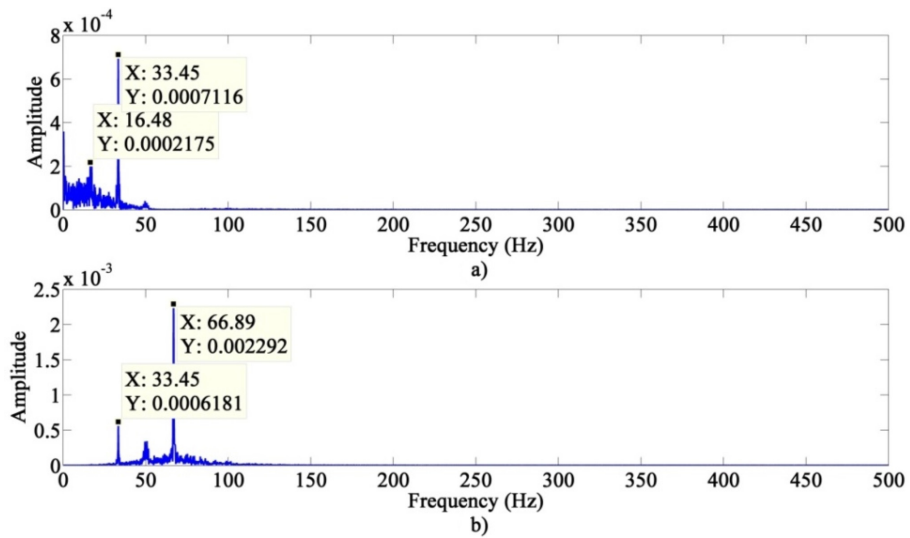

Fig. 14. The power spectrum of the extracted signals after VMD + KICA

Both the frequency of $33.45 \mathrm{~Hz}$ and the frequency of $16.48 \mathrm{~Hz}$ which is equal to half of $f_{r}$ can be found in Fig. 14(a). It can be considered as the feature of rub-impact fault. In Fig. 14(b), the amplitude of $66.89 \mathrm{~Hz}$ is larger than $33.45 \mathrm{~Hz}$, which can be considered as the feature of misalignment fault. Furthermore, the fault features are significantly obvious. It demonstrates that the proposed method has a good performance of the fault feature extraction of rotary shaft with the misalignment fault and rub-impact fault mixed. 


\section{Conclusions}

The method of VMD-based KICA has been proposed to improve the fault feature extraction of single-channel vibration signal of rotary machinery in this paper. In the experiment of rolling bearing with the fault of inner ring and outer ring mixed, the evaluation index $\rho$ of the proposed method is much bigger than wavelets and EEMD+FastICA, it proves that the proposed method makes the fault features more obvious. In the comprehensive experiment with faulty rolling bearing and faulty gear, evaluation index $\rho$ demonstrates that the proposed method can clearly extract the fault feature of complex machinery. The experiment of faulty rotary shaft verifies the effectiveness of this method. Therefore, the proposed method is efficient for fault feature extraction of single-channel vibration signal of rotary machinery.

\section{Acknowledgements}

This research is subsidized by the Natural Science Foundation of China "Research on reliability theory and method of total fatigue life for large complex mechanical structures" (Grant No. U1708255).

\section{References}

[1] Medoued A., Mordjaoui M., Soufi Y., Sayad D. Induction machine bearing fault diagnosis based on the axial vibration analytic signal. International Journal of Hydrogen Energy, Vol. 41, Issue 29, 2016, p. $12688-12695$.

[2] Zuber N., Bajrić R., Šostakov R. Gearbox faults identification using vibration signal analysis and artificial intelligence methods. Physiology and Behavior, Vol. 54, Issue 2, 2014, p. 407-409.

[3] Harmouche J., Delpha C., Diallo D. Improved fault diagnosis of ball bearings based on the global spectrum of vibration signals. IEEE Transactions on Energy Conversion, Vol. 30, Issue 1, 2015, p. 376-383.

[4] Yan R., Gao R. X., Chen X. Wavelets for fault diagnosis of rotary machines: A review with applications. Signal Processing, Vol. 96, Issue 5, 2014, p. 1-15.

[5] Mishra C., Samantaray A. K., Chakraborty G. Rolling element bearing fault diagnosis under slow speed operation using wavelet de-noising. Measurement, Vol. 103, 2017, p. 77-86.

[6] Jiang S. F., Chen Z. G., Shen Q. H., Wu M. H., Ma S. L. Damage detection and locating based on EEMD-Fast ICA. Journal of Vibration and Shock, Vol. 35, Issue 1, 2016, p. 203-209.

[7] Zhao J., Jia R., Wu H. Extraction of vibration signal features based on FastICA-EEMD. Journal of Hydroelectric Engineering, Vol. 36, Issue 3, 2017, p. 63-70.

[8] Xue X., Zhou J., Xu Y., Zhu W., Li C. An adaptively fast ensemble empirical mode decomposition method and its applications to rolling element bearing fault diagnosis. Mechanical Systems and Signal Processing, Vol. 62, 2015, p. 444-459.

[9] Dragomiretskiy K., Zosso D. Variational mode decomposition. IEEE Transactions on Signal Processing, Vol. 62, Issue 3, 2014, p. 531-544.

[10] Mohanty S., Gupta K. K., Raju K. S. Hurst based vibro-acoustic feature extraction of bearing using EMD and VMD. Measurement, Vol. 117, 2018, p. 200-220.

[11] Dermoune A., Wei T. FastICA algorithm: five criteria for the optimal choice of the nonlinearity function. IEEE Transactions on Signal Processing, Vol. 61, Issue 8, 2013, p. 2078-2087.

[12] Grueiro M. Q., Prieto-Moreno A., Santiago O. L. A procedure to configure the FastICA algorithm in fault diagnosis on industrial systems. Ingeniería Electrónica Automática Y Comunicaciones, Vol. 35, Issue 2, 2014, p. 73-89.

[13] Hao T., Tang L. W., Tian G., Zhang Y. Fault diagnosis of gearbox based on KICA. Journal of Vibration and Shock, Vol. 28, Issue 5, 2009, p. 163-167.

[14] Zhang Y., Du W., Li X. Observation and detection for a class of industrial systems. IEEE Transactions on Industrial Electronics, Vol. 64, Issue 8, 2017, p. 6724-6731. 

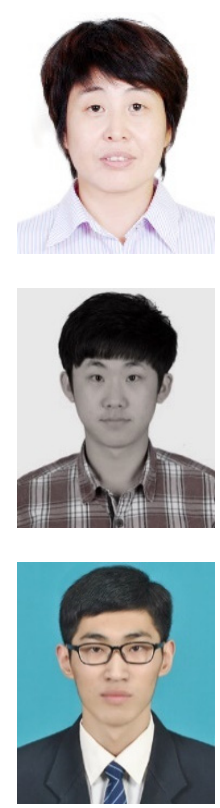

Hongchun Sun received the Ph.D. degree in mechanical engineering from Northeastern University, China. She is currently an Associate Professor with the School of Mechanical Engineering and Automation, China. Her research interests include fault diagnosis, signal processing and nondestructive testing.

Liang Fang received the B.S. degrees in mechanical engineering and automation from Northeastern University in 2015, where he is currently pursuing the M.S. degree in mechanical engineering and automation. His research interests include fault diagnosis and signal processing.

Feng Zhao received the B.S. degrees in School of Mechanical Engineering from Shandong University of Technology in 2017 , and he is currently pursuing the M.S. degrees in mechanical engineering and automation. His research interests include fault diagnosis and image processing. 